\title{
Editorial
}

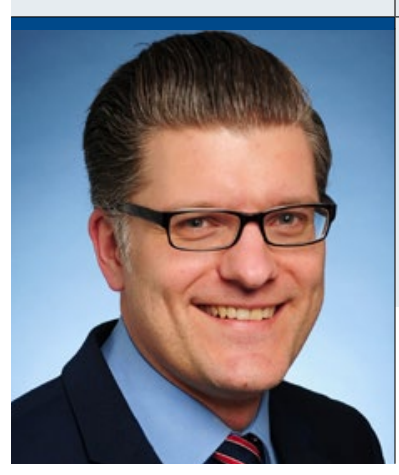

"Grundkenntnisse über Laser und ihre physikalischen

Eigenschaften sind für den Anwender von großer Bedeutung,

da er hieraus die Anwendungsmöglichkeiten der einzelnen

Laser ableiten kann."

\section{Alles Laser, oder was?}

$\mathrm{n}$ einer aktuell onkologisch dominierten Urologie, in der über Immuntherapien, Rezeptorenblocker oder die passende Sequenzbehandlung diskutiert wird, stellt die Endourologie ein wissenschaftliches Mauerblümchen dar, obwohl Harnsteine und das benigne Prostatasyndrom (BPS) das Butter- und Brotgeschäft einer jeden urologischen Klinik darstellen - und dies auch zukünftig so bleiben wird: sei es durch die überalternde Gesellschaft oder durch Migration aus Regionen, die eine höhere Harnsteinprävalenz aufweisen.

\section{Früher verrufen, heute fest etabliert}

Spricht man über eine individuelle maßgeschneiderte, minimal-invasive Harnstein- oder BPS-Therapie, dann führt kein Weg an der Laserstrahlung vorbei. Grundkenntnisse über Laser und ihre physikalischen Eigenschaften sind für den Anwender von großer Bedeutung, da er hieraus die Anwendungsmöglichkeiten der einzelnen Laser ableiten kann. Man erstaunt doch manchmal wie wenig Kenntnis über die Physik von Laserstrahlung tatsächlich vorhanden ist. So ist der Satz „Ich nehme dann mal den Thulium-Laser für die HoLEP“, der mehrfach auf wissenschaftlichen Veranstaltungen vernommen wurde, eindrücklich in Erinnerung geblieben.

Bedingt durch ihre unkritische Anwendung, beispielsweise des Nd:YAG-, FREDDY- oder DYE-Lasers, in den 1980er-Jahren, haben sich Laserverfahren, insbesondere bei der Therapie des BPS, einen schlechten Ruf erworben. Frei nach dem Motto „Ist der Ruf erst ruiniert, lebt es sich ganz ungeniert" hat sich das Bild jedoch grundlegend gewandelt. Der Holmium:YAG-Laser ist aus der modernen BPSund Harnsteintherapie nicht mehr wegzudenken, ebenso der Thulium- und GreenLight-Laser aus der Behandlung des BPS. Insbesondere bei der Enukleation großer Prostataadenome spielen Laserverfahren ihre Stärken hinsichtlich der perioperativen
Morbidität voll aus, auch wenn einige Stimmen noch immer, möglichweise durch DRG-Erlöse getriggert, argumentieren: „Die offene Adenomektomie gehört zur Ausbildung des Urologen dazu“ oder „Für die offene Adenomektomie brauche ich $20 \mathrm{Mi}$ nuten“. Nichtsdestotrotz belegen die AOK-Daten zur BPS-Therapie, dass die stark morbiditätsbehaftete Adenomektomie trotz minimal-invasiver Alternativen auch 2017 nicht totzukriegen ist. Umgekehrt würde heute kein Urologe auf die Idee kommen, einen distalen Harnleiterstein über eine Ureterotomie zu entfernen, „weil die Ureterotomie eben seit jeher zur Ausbildung des Urologen gehört“.

\section{Laser kompakt}

In dieser Dezemberausgabe der URO-NEWS versuchen wir, Ihnen einen Überblick über die physikalischen Grundlagen von Lasern und deren Anwendung zu geben. Neben einem Grundlagenartikel von Andreas J. Gross, der auf die physikalischen Eigenschaften der verschiedenen urologisch genutzten Laser und die sich daraus ableitenden Einsatzmöglichkeiten eingeht, runden Artikel zur Anwendung von Lasern in der Harnsteintherapie von Armin Secker, bei der BPS von Christopher Netsch et al. sowie bei Harnblasenkarzinomen von Julian Struck et al. den Schwerpunkt ab.

Beim Lesen dieser Laser-Ausgabe der URONEWS wünsche ich Ihnen viel Spaß,

Ihr

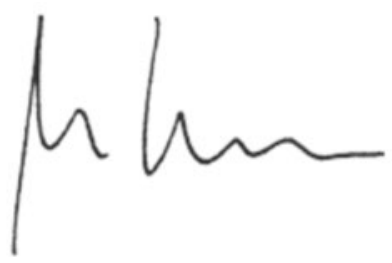

